\title{
ON THE GEOMETRY OF NONCOMMUTATIVE SPECTRAL THEORY
}

\author{
BY ERIK M. ALFSEN AND FREDERIC W. SHULTZ
}

Communicated by P. R. Halmos, March 24, 1975

We shall consider an order unit space $(A, e)$ and a base-norm space $(V, K)$ in separating order and norm duality with $A$ pointwise monotone $\sigma$-complete, i.e. for every descending sequence $\left\{a_{n}\right\}$ in $A^{+}$there exists $a \in A$ such that $\langle a, x\rangle=$ $\lim _{n}\left\langle a_{n}, x\right\rangle$ for $x \in K$. (See [1] for definitions and proofs.) We write $M \in T_{A}$ if $M$ is a weakly closed supporting hyperplane of $A^{+}$and $\widetilde{F}=\bigcap\left\{M \in T_{A} \mid F \subset M\right\}$ for $F \subset A^{+}$. (One may think of $\widetilde{F}$ as a "minimal tangent space" for $A^{+}$at $F$.) $M$ is a smooth order ideal of $A$ if $M=\left(A^{+} \cap M\right)^{\sim}$, and $F$ is a semiexposed face of $A^{+}$if $F=A^{+} \cap \widetilde{F}$. For a projection $P: A \rightarrow A$ we write im ${ }^{+} P=A^{+} \cap \operatorname{im} P$, $\mathrm{ker}^{+} P=A^{+} \cap \operatorname{ker} P$. Two projections $P, Q: A \rightarrow A$ are quasi-complementary (q.c) if $\mathrm{im}^{+} Q=\mathrm{ker}^{+} P, \mathrm{ker}^{+} Q=\mathrm{im}^{+} P$. Similar definitions apply with $V$ in place of $A$. A weakly continuous positive projection $P$ of $A$ (or $V$ ) with $\|P\| \leqslant 1$ is smooth if $\operatorname{ker} P$ is a smooth order ideal. A projection $R$ of $V$ is neutral if $\|R v\|$ $=\|v\|$ implies $R v=v$ for $v \in V^{+}$. This term relates to physical filters which are "neutral" in that when a beam passes through with intensity undiminished (\|Rv\| $=\|v\|)$, then the filter is neutral to it $(R v=v)$.

THEOREM 1. For projections on $A$ the following are equivalent: (i) $P, Q$ are q.c. and so are the dual projections $P^{*}, Q^{*}$; (ii) $P, Q$ are q.c. and both are smooth; (iii) $P^{*}, Q^{*}$ are q.c. and both are smooth; (iv) $P, Q$ are q.c. and $P^{*}, Q^{*}$ are neutral.

$P: A \rightarrow A$ is a $P$-projection (in symbols $P \in P$ ) if it admits a (necessarily unique) q.c. $P^{\prime}=Q$ such that (i)-(iv) hold. To $P \in P$ we associate a projective unit $u=P e \in A(u \in U)$ and a projective face $F=K \cap \operatorname{im} P^{*}$ of $K(F \in F)$. We write $F_{P}=K \cap \operatorname{im} P^{*}$ and $F_{P}^{\#}=F_{P^{\prime}}$. Now $P, U, F$ are in natural 1-1 correspondence. $P \in P$ is compatible with $a \in A$ if $a=P a+P^{\prime} a$; when $a=Q e$ with $Q \in P$, this will hold iff $P, Q \in P$ commute, then we say $P, Q$ are compatible. Also we say $P \in P$ is bicompatible with $a \in A$ if $P$ is compatible with $a$ and with all $Q \in P$ compatible with $a$. An affine retraction $\rho: K \rightarrow K^{\prime} \subset K$ is said to be transversal at $F \subset K^{\prime}$ if $\rho(y)=\rho(z)$ implies $y-z \in \widetilde{F}$.

AMS (MOS) subject classifications (1970). Primary 46A40, 46L05, 46L10, 47 A60.

Key words and phrases. $C^{*}$-algebras, von Neumann algebras, state spaces, compact convex sets, ordered linear spaces, simplexes, spectral theory, functional calculus, noncommutative. 
THEOREM 2. Let $F, G \subset K$ and suppose $A \simeq V^{*}$. Then $F, G$ are q.c. projective faces of $K$ iff: (i) $F$ and $G$ are semiexposed faces, (ii) $F$ and $G$ are affinely independent, and (iii) there is an affine retraction $\rho: K \rightarrow \operatorname{co}(F \cup G)$, transversal at $F$ and $G$. If (i)-(iii) hold and $F=F_{P}$, then $\rho=\left.\left(P+P^{\prime}\right)^{*}\right|_{K}$ is the unique affine retraction of $K$ onto $\operatorname{co}(F \cup G) . F$ is a split face of $K$ iff $P$ is compatible with all $a \in A$ (then $\rho=\mathrm{id})$.

Now we shall impose an axiom similar to the one used in (commutative) integration theory by Stone to connect the linear functional approach with measure theory [3]: $A$ and $V$ are said to be in weak spectral duality if for every $a \in A$ and $\lambda \in \mathbf{R}$ there exists a projective face $F$ compatible with $a$ such that $\langle a, x\rangle \leqslant \lambda$ for $x \in F$ and $\langle a, x\rangle\rangle \lambda$ for $x \in F^{\#}$. If, in addition, $F$ is unique, then we say that $A$ and $V$ are in spectral duality. The requirement of uniqueness for $F$ above is equivalent to the requirement that $F$ be bicompatible with $a$. We always have the implications: spectral $\Rightarrow$ weakly spectral $\Rightarrow$ all exposed faces are projective. In finite dimensions the reverse implications hold. In infinite dimensions the second implication cannot be reversed; whether weakly spectral implies spectral remains an open question. Henceforth we assume $A$ and $V$ are in spectral duality. (For Theorem 3 projectivity of exposed faces would suffice, and for the existence part of Theorem 4 weak spectral duality would suffice.)

THEOREM 3. The set $U$ of projective units is a $\sigma$-complete orthomodular lattice.

THEOREM 4. For every $a \in A$ there exists a unique spectral family $\left\{e_{\lambda}\right\}$ in $U$ (with customary properties) such that $\langle a, x\rangle=\int \lambda d\left\langle e_{\lambda}, x\right\rangle(x \in K)$.

We denote by $B$ the bounded Borel functions on $\mathbf{R}$ and by $\boldsymbol{\gamma}$ the function $\gamma(\lambda)=\lambda$ for $\lambda \in \mathbf{R}$. A map $\Phi: B \rightarrow A$ is said to be a morphism if $\Phi$ is linear and positive, if $\Phi(1)=e$, and if $\varphi_{n} \downarrow 0$ (pointwise on R) implies $\Phi\left(\varphi_{n}\right) \downarrow 0$ (pointwise on $K$ ); also $\Phi$ is said to be extreme point preserving if $\Phi\left(\chi_{E}\right)$ is an extreme point of $[0, e]=\{a \in A \mid 0 \leqslant a \leqslant e\}$ for every Borel set $E \subset \mathbf{R}$. (The extreme points of $[0, e]$ are the projective units.) For $a \in A$ and $\varphi \in B$ we define $\varphi(a) \in A$ by $\langle\varphi(a), x\rangle=\int \varphi(\lambda) d\left\langle e_{\lambda}, x\right\rangle$ for $x \in K$.

TheOREM 5. For $a \in A$ the map $\varphi \rightarrow \varphi(a)$ is the unique extreme point preserving morphism of $B$ into $A$ such that $\gamma \rightarrow a$; if $\varphi, \psi \in B$, then $(\varphi \circ \psi)(a)=$ $\varphi(\psi(a))$.

Let now $K$ be any convex compact set and $A(K)$ (resp. $A^{b}(K)$ ) the continuous (bounded) affine functions on $K$. We say $K$ is spectral if $V=A(K)^{*}$ and $A=$ $V^{*} \simeq A^{b}(K)$ are in spectral duality, and strongly spectral if, in addition, all $e_{\lambda}$ in the spectral family of each $a \in A(K)$ are u.s.c. on $K$. $K$ is strongly spectral iff $A(K) \subset A^{b}(K)$ is closed under functional calculus by continuous functions. The unit balls of $L^{p}$ for $1<p<\infty$ are strongly spectral. Every Choquet simplex is 
spectral, and it is strongly spectral iff it is a Bauer simplex. If $\mathscr{Q}$ is a von Neumann algebra with identity $e$, predual $\mathfrak{Q}_{*}$, and normal state space $K$, then $\left(\mathfrak{U}_{s a}, e\right)$ and $\left(\left(\mathscr{U}_{*}\right)_{s a}, K\right)$ are in spectral duality. Here $P: A \rightarrow A$ is a P-projection iff $P a=$ pap for some projection $p \in \mathbb{Q}$ and all $a \in A$, the projective units are the projections $P e=p e p=p \in \mathcal{U}$, and the projective faces $F$ of $K$ are the norm closed ones. If I is $a C^{*}$-algebra with identity, then the state space $K$ is strongly spectral. Generally our concepts here coincide with similarly named concepts in operator theory (e.g. the spectral theorem and functional calculus), and we obtain generalizations and new (more "geometric") proofs of various results in operator theory (e.g. that the normalized traces of a $C^{*}$-algebra with identity form a $w^{*}$-compact simplex $[2],[4])$.

\section{REFERENCES}

1. E. M. Alfsen and F. W. Shultz, Non-commutative spectral theory for affine function spaces on convex sets, Oslo University, 1974/75 (preprint).

2. E. G. Effros and F. Hahn, Locally compact transformation groups and $C^{*}$-algebras, Mem. Amer. Math. Soc. No. 75 (1967). MR 37 \#295.

3. M. H. Stone, Notes on integration. I, II, Proc. Nat. Acad. Sci. U.S.A. 34 (1948), 336-342, 447-455. MR 10, 24, 107.

4. E. Thoma, Über unitäre Darstellungen abzahlbärer, diskreter Gruppen, Math. Ann. 153 (1964), 111-138. MR 28 \#3332. WAY

MATHEMATICS INSTITUTE, UNIVERSITY OF OSLO, BLINDERN, OSLO 3, NOR-

DEPARTMENT OF MATHEMATICS, WELLESLEY COLLEGE, WELLESLEY, MASSACHUSETTS 02181 